Pacific Journal of Mathematic 


\title{
REMARKS ON PIECEWISE-LINEAR ALGEBRA
}

\author{
EDUARDo D. SonTAG
}

\begin{abstract}
This note studies some of the basic properties of the category whose objects are finite unions of (open and closed) polyhedra and whose morphisms are (not necessarily continuous) piecewise-linear maps.
\end{abstract}

Introduction. A function $f: V \rightarrow W$ between real vector spaces is piecewise-linear (PL) if there exists a partition of $V$ into "open polyhedra" $X_{i}$ (i.e., relative interiors of polyhedra) such that $f$ is affine on each $X_{i}$. (As distinct to the case of PL-topology, no continuity is required of $f$.) Images and preimages under PL-maps give rise to finite unions of open polyhedra, or PL-sets; conversely PL functions can be characterized by the fact that their graphs are PL-sets. This paper studies some basic algebraic properties of the category $\boldsymbol{P L}$, proving in particular that it is an exact category, and in fact a pretopos. A classification is given for the isomorphism classes of objects of $\boldsymbol{P L}$, in terms of a two-generator semiring.

The first section recalls without proof some facts from polyhedral geometry needed in the paper. Except for the setting of a unified notation and for minor generalizations, the material there is well known. The second section defines PL maps and sets, and studies the category. The main results (leading to the classification theorem) are given in the last section.

1. Review of polyhedral geometry. The following conventions and definitions hold throughout. All vector spaces are finitedimensional spaces over the reals $\boldsymbol{R}$; a flat means an affine submanifold of some such space $V$, and the closed half-spaces associated to a linear $f: V \rightarrow \boldsymbol{R}$ and an $\boldsymbol{r}$ in $\boldsymbol{R}$ (or associated to the hyperplane $\{x \mid f(x)=r\}$,) are the sets $\{x \mid f(x) \leqq r\}$ and $\{x \mid f(x) \geqq r\}$. The corresponding open half-spaces are obtained by using strict inequalities in the above. A half-line (closed or open) is the intersection of a line $L$ in $V$ with a (closed or open) half-space not containing $L$.

A (convex) closed polyhedron in $V$ is by definition an intersection of finitely many closed half-spaces. The dimension of a nonempty polyhedron $P$ is the dimension of aff $(P)$, the smallest flat containing $P$; the relative interior $\mathrm{ri}(P)$ is the interior of $P$ relative to the usual topology on aff $(P)$. An open polyhedron $P$ is by definition the relative interior of some closed polyhedron $c \ell(P)$ (c $\ell$ denoting 
usual topological closure); this is equivalent to $P$ being an intersection of finitely many flats and open half-spaces. A (closed or open) polytope is a polyhedron which is bounded (=contains no half-lines). The relative boundary $\mathrm{rb}(P)$ is the set-theoretic difference $c \ell(P) \backslash \mathrm{ri}(P)$.

We review now a few other notions and results which are needed later; for proofs the reader is referred to Rockaffelar [7], especially $\S \S 8,9,17,18$, and 19, and to Grünbaum [5]. (The terminology "polyhedron" is usually reserved for closed ones and results are stated for these; results for "open polyhedra" are implicit in results about relative interiors. In our case, open polyhedra will be more relevant.)

A proper ("exposed") face of a closed polyhedron $P$ (or, more generally, of a convex set,) is the intersection of $P$ with a nontrivial supporting hyperplane $H$ (i.e., an $H$ such that $H$ intersects $P$ and $P$ is contained entirely in one of the half-spaces associated to $H$ ). A face of $P$ is either $P$ itself, the empty set, or a proper face. A zero-dimensional, one-dimensional, or maximal proper face is called respectively a vertex (or extreme point), edge, or facet. An extreme direction (or "extreme point at infinity") is the direction of a halfline contained in some edge (by direction one means a translationequivalence class of half-lines $\operatorname{dir}(y)=$ family of sets $\{x+\lambda y, \lambda \geqq 0\}$, $x$ in $V$, for a nonzero vector $y$ ).

If $P$ is an irredundant intersection of half-spaces each associated to a hyperplane $H_{i}$, the facets of $P$ are precisely the intersections of $P$ with the various $H_{i}$. The facets of facets of $P$ are intersections of facets of $P$, and every face of $P$ is an intersection of facets. The set $F(P)$ of faces of $P$, ordered by inclusion, is a complete lattice, the meet being the intersection. The following result (see e.g., Rockaffelar [7, Theorem 18.2]) will be useful.

THEOREM 1.1. The family of relative interiors $\mathrm{ri}(F), F$ in $F(P)$, gives a partition of $P$.

Note that the $\operatorname{ri}(F)$ for $F$ proper give a partition of $\operatorname{rb}(P)$.

A face of an open polyhedron $P$ is by definition the same thing as a face of $c \ell(P)$.

A (closed or open) polyhedron $P$ recedes in direction $d=\operatorname{dir}(y)$ if $x+\lambda y$ is in $P$ whenever $x$ is in $P$ and $\lambda$ is nonnegative. This is equivalent to requiring that there exist at least one $x$ in $P$ with $\{x+\lambda y, \lambda \geqq 0\}$ contained in $P$. The recession cone $0^{+} P$ is the set consisting of zero and of all vectors $y$ such that $\operatorname{dir}(y)$ is a direction of recession of $P$, or equivalently, the set of $y$ with $P+y \subseteq P$. (Here $C+y$, or more generally $A+B$, indicates as usual the set of all sums.) For any $P$, it holds that $0^{+} P=0^{+}(\operatorname{ri}(P))$. 
The (closed) convex hull conv (S) of a finite subset $S$ of $V$ is the smallest convex subset of $V$ containing $S$; it is the closed polyhedron consisting of all convex combinations $\sum \lambda_{i} s_{i}, \sum \lambda_{i}=1, \lambda_{i} \geqq 0$, of elements of $S$. The open convex hull opconv $(S)$ is obtained by restricting to $\lambda_{i}>0$ in the above; this is the same as ri (conv $\left.(S)\right)$. If $D$ is a set of directions of $V, \operatorname{ray}(D)$ is the union of zero and the set of all vectors $y$ whose directions belong to $D$. For a finite set of points $S$ and directions $D$, conv $(S, D)$ is the smallest convex set containing $S$ and receding in all directions of $D$, i.e., conv $(S+\operatorname{ray}(D))$. The latter is also equal to $\operatorname{conv}(S)+$ cone $(D)$, where cone $(D)=$ conv (ray $(D))$; thus conv $(S, D)$ is the set of all sums $\sum \lambda_{i} x_{i}+\sum \mu_{j} y_{j}$, where the $x_{i}$ are in $S$, the $y_{j}$ are arbitrary vectors with directions in $D$, and the $\lambda_{i}, \mu_{j}$ are nonnegative with $\sum \lambda_{i}=1$. The corresponding open convex hull opconv $(S, D)=\operatorname{ri}(\operatorname{conv}(S, D))$ is obtained by restricting the $\lambda_{i}, \mu_{j}$ to be strictly positive. One of the basic results on polyhedra, due to Minkowski and Weyl, can now be stated (see Rockaffelar [7, Theorem 19.1]):

THEOREM 1.2. The following are equivalent:

(a) $P$ is a (closed) polyhedron;

(b) $P$ is a closed convex set with finitely many faces;

(c) $P$ is finitely generated, i.e., $P=\operatorname{conv}(S, D)$ for some finite $S, D$.

Further, if $P$ is a line-free polyhedron, then $S$ above [resp., D] can be chosen as the set of extreme points [resp., extreme directions,] of $P$.

Polyhedral sets are preserved by images and preimages under linear transformations; this is clear from (c) above. But the larger class of projective transformations is also compatible with the polyhedral structure. A projective transformation is obtained by embedding the given affine space into a projective space and then restricting to a different affine open set. In local coordinates, say for $V=\boldsymbol{R}^{n}$, this process induces a partial map of the form

$$
x \longmapsto(\langle c, x\rangle+d)^{-1}(A x+b),
$$

where $A$ is linear and $\|c\|^{2}+d^{2} \neq 0$. Grünbaum's book discusses these transformations in detail. Projective transformations preserve colinearity and the polyhedral sets in their domain. Questions about polyhedra can then be reduced to the case of polytopes. For example, consider the nonsingular projective transformation:

$$
\alpha: x \longmapsto\left(1+x_{1}+\cdots+x_{n}\right)^{-1}\left(x_{1}, \cdots, x_{n}\right) .
$$

This maps the open positive orthant $\boldsymbol{R}_{+}^{n}$ of $\boldsymbol{R}^{n}$ into the following 
open polytope $\Delta_{n}$ of $\boldsymbol{R}^{n}$,

$$
x_{1}+\cdots+x_{n}<1, \quad x_{i} \geqq 0,
$$

in such a way that directions in $\boldsymbol{R}_{+}^{n}$ become identified to just points in $x_{1}+\cdots+x_{n}=1, x_{i} \geqq 0$. Open polyhedra $P$ in $\boldsymbol{R}_{+}^{n}$ correspond to open polytopes $Q$ contained in $\Delta_{n}$, extreme points [resp., extreme directions] of $P$ corresponding to extreme points of $Q$ [resp., extreme points of $Q$ lying in $x_{1}+\cdots+x_{n}=1$ ].

A (generalized) $m$-simplex is the (closed or open) convex hull of $m+1$ affinely independent points and directions, i.e., of a set $S \cup D$ of cardinality $m+1$ for which aff $(\operatorname{conv}(S, D))$ has dimension $m$. Since projective equivalences preserve affine independence in their domains, simplexes correspond to simplexes under (1.3), and the usual (polytope) baricentric subdivision theorem implies, (after if necessary subdividing a given polyhedron into its intersection with orthants,):

LEMMA 1.5. Every (open or closed) polyhedron is a disjoint union of open simplexes.

This lemma will be useful in the last section.

\section{PL-sets and maps.}

Definition 2.1. The PL-subsets of a vector space $V$ are those belonging to the Boolean algebra $\mathrm{PL}(V)$ generated by all the open (or all the closed) half-spaces of $V$. A PL-set is a PL-subset of some $V$. A PL-relation $R: X \rightarrow Y$ is one which is a PL-set as a subset of $X \times Y$; a PL-map $f: X \rightarrow Y$ is a map which is a PL-relation.

A PL-set is thus the same as a disjoint union of open polyhedra. A number of facts are therefore immediate consequences of those known for polyhedra. For example, images and preimages under linear maps preserve PL-sets, and the product $X \times Y$ of PL-subsets of $V, W$ is a PL-subset of $V \times W$.

LEMma 2.2. Let $R: X_{1} \rightarrow X_{2}$ and $S: X_{2} \rightarrow X_{3}$ be PL-relations, with the $X_{i}$ in $\mathrm{PL}\left(V_{i}\right)$. Then

$$
R \& S:=\{(x, y, z) \text { in } X \times Y \times Z \mid x R y \text { and } y S z\}
$$

is a PL-set.

Proof. Just note that $R \& S=\left(R \times V_{3}\right) \cap\left(V_{1} \times S\right)$.

Projection of the above on $V_{1}$ and $V_{3}$ yields:

CoROLlaRY 2.3. A composition of PL-relations is a PL-relation. 
Thus PL-maps are also closed under composition, and we have a well-defined category $\boldsymbol{P L}$. Note that since PL-maps are defined through their graphs, $f^{-1}$ is a PL-relation for any PL-map $f$, and therefore isomorphisms in $\boldsymbol{P L}$ are the same as PL-bijections. Before studying general properties of $\boldsymbol{P L}$ we need several technical facts. A PL-map is a "piecewise linear" map in the following sense.

Lemma 2.4. If $f: X \rightarrow Y, X$ is in $\mathrm{PL}(V), Y$ is in $\mathrm{PL}(W)$, and $X=\cup X_{i}$ is a finite covering such that each restriction $f \mid X_{i}$ is a PL-map, then $f$ is a PL-map. Conversely, assume that $f: X \rightarrow Y$ is a PL-map and let $X=\cup X_{i}^{\prime}$ and $Y=\cup Y_{j}^{\prime}$ be (finite) partitions into open polyhedra. Then there exist (finite) open polyhedral partitions $X=\cup X_{i}$ and $Y=\cup Y_{j}$ which refine the original ones and such that each restriction $f \mid X_{i}$ is the restriction of a affine map $V \rightarrow W$, and maps $X_{i}$ into some $Y_{j}$. Further, if $f$ is one-to-one, then there exist simultaneous open refinements as above $X=\cup X_{i}, f(X)=\cup Y_{i}$, with $f\left(X_{i}\right)=Y_{i}$ and each $f \mid X_{i}$ the restriction of an invertible linear map from aff $\left(X_{i}\right)$ onto aff $\left(Y_{i}\right)$.

Proof. The first assertion is clear from the fact that (the graph of) $f$ is the union of (the graphs of) the $f \mid X_{i}$.

To prove the remaining assertions, let $X=\cup X_{i}^{\prime}, Y=\cup Y_{j}^{\prime}$, and $G=\cup G_{k}$ be partitions of $X, Y$, and $G=$ graph of $f$, into open polyhedra. Let $G_{i j k}$ be the intersection of $G_{k}, p r_{1}^{-1}\left(X_{i}^{\prime}\right)$, and $p r_{2}^{-1}\left(Y_{j}^{\prime}\right)$, where $p r_{i}$ denotes projection of $V \times W$ into the $i$ th factor. Each $G_{i j k}$ is an open polyhedron and projects into $X_{i}^{\prime}, Y_{j}^{\prime}$. Since $f$ is a function, the family of all $p r_{1}\left(G_{i j k}\right)$ is a partition of $X$ into open polyhedra $X_{i j k}$, which refines the original one; note that $X_{i j k}$ maps into $Y_{j}^{\prime}$. When $f$ is one-to-one, the $Y_{i j k}:=p r_{2}\left(G_{i j k}\right)$ are disjoint, and $f\left(X_{i j k}\right)=Y_{i j k}$. The last statement follows from the fact that the projections restricted to aff $\left(G_{i j k}\right)$ establish isomorphisms with aff $\left(X_{i j k}\right)$, aff $\left(Y_{i j k}\right)$, since they are one-to-one on the open subset $G_{i j k}$ of aff $\left(G_{i j k}\right)$.

A PL-map is in fact, up to PL-automorphisms of its domain, a linear map, since $X \cong$ graph $(f)$. This fact is itself not too useful, (since the automorphism carries all the nonlinearity,) but is implicit in arguments like the above. (The terminology "PL" should not be confused with the very different notion that appears in combinatorial topology, where all (PL) maps are continuous and all polyhedra are closed polytopes.)

A general way of constructing PL-sets is the following. Let $L$ be the first-order language over the alphabet having: constants $r$ for each real number $r$, variables $x_{1}, \cdots, x_{n}, \cdots$, unary function 
symbols " $r$.( )" for each $r$ real, binary function symbol + , and relational symbols $>$, $=$.

LemMA 2.5. Every sentence $S$ in $L$ defines a PL-set, i.e., if $x_{1}, \cdots, x_{n}$ are the free variables in $S$ then

$$
\operatorname{Dom}(S):=\left\{\left(x_{1}, \cdots, x_{n}\right) \text { in } \boldsymbol{R}^{n} \mid S\left(x_{1}, \cdots, x_{n}\right)\right\}
$$

is a PL-set. Conversely, any PL-subset of an $\boldsymbol{R}^{n}$ can be defined in this fashion.

Proof. The converse part is clear, since every open half-space, or hyperplane, can be defined by an equation $a_{1} x_{1}+\cdots+a_{n} x_{n}<r$, or $=r$. To prove that $\operatorname{Dom}(S)$ is always a PL-set, it is enough to prove that all atomic formulas define PL-sets and that closing under $\neg, \wedge, \exists$, preserves the PL-structure. But atomic formulas are all linear equations or inequalities, so they define hyperplanes or halfspaces, and they are therefore PL-sets. Closure under negation and conjunction holds by the Boolean closure (complements, intersections). Finally, $\{x \mid(\exists y) S(x, y)\}$ is the projection of $\{(x, y) \mid S(x, y)\}$; it is therefore $\mathrm{PL}$ if the latter is.

Another way of expressing the conclusion of the above lemma is by saying that any set defined using existential or universal quantifiers can be also defined using only propositional connectives. In using the above one usually extends the language (informally) to include sentences containing PL-functions (since these are defined by their graphs, which are PL-sets), and arbitrary PL-sets (since under isomorphism a $P$ in $\mathrm{PL}(V)$ is a subset of some $\boldsymbol{R}^{n}$ ). Similarly, one can bound quantifiers, as in "for all $x$ in $S, \ldots$ ", when $S$ is known to be a PL-set.

For example, consider the situation in applications in which one has a family of PL-maps

$$
f_{y}=f(\cdot, y): X \longrightarrow Z, \quad y \text { in } Y,
$$

where $f: X \times Y \rightarrow Z$ is a fixed PL-map. Here it is natural to ask whether the sets of the $y$ in $Y$ where $f$ is one-to-one, or onto, are PL-sets. In view of 2.5, the answer is (trivially) yes: for instance, $f$ is one-to-one for the $y$ in:

$$
\left\{y \text { in } Y \mid f(x, y)=f\left(x^{\prime}, y\right) \text { implies } x=x^{\prime}\right\},
$$

which is expressable as a first-order sentence. Similarly, given a $z_{0}$ in $Z$, the set of common "zeros"

$$
\left\{x \text { in } X \mid f_{y}(x)=z_{0} \text { for all } y\right\}
$$


is a PL-set. Related to this is the set of all $x$ for which there is some $y$ solving the equations:

$$
X_{0}:=\left\{x \text { in } X \mid \text { there exists } y \text { with } f(x, y)=z_{0}\right\},
$$

which is again PL. One of the most useful properties of the category $\boldsymbol{P L}$ is the validity of the following global implicit function theorem:

THeOREM 2.10. Let $f: X \times Y \rightarrow Z$ be a PL-map, $z_{0}$ in $Z$, and $X_{0}$ as in (2.9). Then there exists a PL-map $s: X_{0} \rightarrow Y$ such that $f(x, s(x))=z_{0}$ for all $x$ in $X_{0}$.

Letting $R$ be the relation " $x R y$ iff $f(x, y)=z_{0}$ ", this becomes a consequence of the first part of the following stronger result:

THEOREM 2.11. Let $R: X \rightarrow Y$ be a PL-relation with domain $X_{0}$. There exists then a PL-map s: $X_{0} \rightarrow Y$ such that:

(a) $s(x)$ is in $R(x)$ for all $x$, and

(b) $R(x)=R\left(x^{\prime}\right)$ implies $s(x)=s\left(x^{\prime}\right)$.

Proof. With a slightly different terminology, the problem is that of showing that for each PL-subset $R$ of $V \times W$ there is a section $s$ of $p r_{1}$ such that $s(x)$ depends only on $p r_{1}^{-1}(x)$. Without loss of generality we assume $V=\boldsymbol{R}^{n}, W=\boldsymbol{R}^{k}$. The result will follow by induction on $k$ once it is established for $k=1$. Indeed, assume it is true for $k$ and let $R$ be a PL-subset of $R^{n+k+1}$. Let $X_{1}$ be the projection of $R$ on the first $n+1$ coordinates. The result being true for $k=1$ means that there is an $s_{1}: X_{0} \rightarrow R$ with $s_{1}(x)$ in $R_{1}(x)$ for all $x$ and $s_{1}(x)=s_{1}\left(x^{\prime}\right)$ if $R_{1}(x)=R_{1}\left(x^{\prime}\right)$, where $R_{1}$ is just $X_{1}$ seen as a relation $\boldsymbol{R}^{n} \rightarrow \boldsymbol{R}$; note that $X_{0}$ is the domain both of $R$ and of $R_{1}$. By the inductive hypothesis there is also an $s_{2}: X_{1} \rightarrow \boldsymbol{R}^{k}$ satisfying (a), (b). The desired $s$ can be then obtained defining $s(x):=$ $s_{2}\left(x, s_{1}(x)\right)$.

Thus the problem reduces to the case $k=1$. Further, we may assume that $R$ is an open polyhedron. If $R$ is a more general PLset we can write $R$ as a union of the disjoint open polyhedra $R_{1}, \cdots, R_{t}$. If for each of these there exists an $s_{i}: p r_{1}\left(R_{i}\right) \rightarrow R$, an $s: p r_{1}(R) \rightarrow R$ can be constructed using 2.4 by defining $s:=s_{i}$ on $X_{i}$, where $X_{1}$ is $\operatorname{pr}_{1}\left(R_{1}\right)$ and $X_{i}$ is $\operatorname{pr}\left(R_{i}\right) \backslash X_{i-1}$ for $i>1$. This $s$ will satisfy both (a), (b).

We divide the case $R=$ open polyhedron in $\boldsymbol{R}^{n} \times \boldsymbol{R}$ into four disjoint cases: (i) the vector

$$
e_{n+1}:=(0, \cdots, 0,1)
$$

is in $0^{+} R$ and $-e_{n+1}$ is not, (ii) $e_{n+1}$ is not in $0^{+} R$ but $-e_{n+1}$ is, (iii) 
both $\pm e_{n+1}$ are in $0^{+} R$, and (iv) neither of $\pm e_{n+1}$ is in $0^{+} R$. Let

$$
\begin{aligned}
& r_{1}(x):=\inf \{r \mid(x, r) \text { is in } R\}, \\
& r_{2}(x):=\sup \{r \mid(x, r) \text { is in } R\}
\end{aligned}
$$

(these may be $\pm \infty$ ). If (i) holds, we define $s(x):=r_{1}(x)+1$; if (ii) holds, $s(x):=r_{2}(x)-1$; if (iii) then $s(x):=0$; and if (iv) then $s(x):=$ $1 / 2\left(r_{1}(x)+r_{2}(x)\right)$. Property (b) is trivially true for such a definition of $s$, since the $r_{i}(x)$ depend only on the corresponding $R(x)$. With respect to (a), case (i) corresponds to the situation in which $(\{x\} \times$ $\boldsymbol{R}) \cap c \ell(R)$ is always a half-line not bounded "above", thus $\left(x, r_{1}(x)\right)+$ $e_{n+1}$ is in $R$, since $\left(x, r_{1}(x)\right)$ is in $c \ell(R)$ and $0^{+} R=0^{+}(c \ell R)$. The same argument applies to case (ii). Case (iii) corresponds to $(\{x\} \times \boldsymbol{R}) \cap c \ell(R)$ being a finite interval with endpoints $\left(x, r_{1}(x)\right)$ and $\left(x, r_{2}(x)\right)$; thus $(x, s(x))$ is in $\operatorname{ri}(c \ell(R))=R$. In case (iv) the entire line $\{x\} \times R$ is in $R$ and thus $(x, 0)$ is in $R$.

It only remains to prove that $s$ is piecewise linear. For this it is enough to show that $r_{1}$ and $r_{2}$ are PL-maps when finite-valued. We work with $r_{1}$; the argument for $r_{2}$ being the same. Let $F_{1}^{0}, \cdots, F_{t}^{0}$ be the relative interiors of the proper faces of $R$. These cover $r b(R)$, so that $r_{1}(x)$ is always in a suitable $F_{j}^{0}$ when finite. We drop from the list all those $F_{j}^{0}$ for which $\{x\} \times \boldsymbol{R}$ either doesn't intersect $F_{j}^{0}$ or intersects $F_{j}^{0}$ at more than one point. Note that, for the latter, $r_{1}(x)$ will be in a face of $F_{j}^{0}$, which is in turn a lowerdimensional $F_{i}^{0}$. Since the projections $p r_{1} \mid F_{j}^{0}$ are now all one-to-one, they admit PL-sections (whose graphs are the $F_{j}^{0}$ themselves). If $r_{1}(x)$ belongs to a certain $F_{j}^{0}$ then also $r_{1}\left(x^{\prime}\right)$ is in the same $F_{j}^{0}$ for any other $x^{\prime}$ in $\operatorname{pr}_{1}\left(F_{j}^{0}\right)$ : otherwise, there is some $\varepsilon>0$ such that

$$
z^{\prime}:=(1+\varepsilon) r_{1}(x)-\varepsilon z
$$

is in $F_{j}^{0}$, where $z$ is the unique point in $F_{j}^{0}$ projecting into $x^{\prime}$, since $F_{j}^{0}$ is relatively open and convex. But then

$$
(1+\varepsilon)^{-1}\left(z^{\prime}+\varepsilon r_{1}\left(x^{\prime}\right)\right)
$$

is in $c \ell(R)$, projects into $x$, and is strictly less than $r_{1}(x)$, contradicting the definition of the latter. Thus $r_{1}$ is a PL-map on each of the (disjoint) $p r_{1}\left(F_{j}^{0}\right)$, and is therefore PL itself.

The above remarks help in characterizing the category $\boldsymbol{P L}$. The terminology "subset" will be used in the set-theoretic sense, while "subobject" will be used in the categorical sense. Note that the category of finite sets is equivalent to a full subcategory of $P L$; the subcategory of finite sets of integers (as PL subsets of $\boldsymbol{R}$ ) gives the representation. By arguments as in Sets, (and using the above 
results,) it is easy to establish that: (i) $0=\varnothing$ and $1=\{0\}$ are initial and final objects respectively; (ii) 0 is a generator for the category, and $\mathbf{2}=\{0,1\}$ is a cogenerator; and (iii) equalizers and finite products and coproducts exist (first order constructions). Thus PL is finitely complete with finite coproducts, with the respective (co)limits preserved by the forgetful functor to Sets. It is equivalent for a PL-map to be a monomorphism, one-to-one, a coretraction, or an equalizer. Similarly, epimorphism, onto, retraction, and coequalizer are equivalent properties. A theory of congruences exists, as with algebraic theories. A congruence on a PL-set $X$ is a PL-relation $R: X \rightarrow X$ which is an equivalence relation. Then one can prove that a relation $R$ is a congruence iff $R=\operatorname{Ker} f=\{(x, y) \mid f(x)=f(y)\}$ for some PL-map $f$ with domain $R$. The induced-homomorphism property holds for congruences. Thus PL is a pretopos (Johnstone [6]).

It follows that $\boldsymbol{P L}$ admits a full, limit-preserving, and finite coproduct-preserving embedding into the geometric topos $\operatorname{shv}(\boldsymbol{P L}, J)$ of sheaves for the precanonical (Grothendieck) topology $J$ on $\boldsymbol{P L}$. This embedding is for example useful in system theory, when one studies the category Mach $(\boldsymbol{P L})$ of machines (see Arbib and Manes [1]) over $\boldsymbol{P L}$. The canonical realization functor does not admit a adjoint over $\boldsymbol{P L}$, but adjoints do exist over the larger category of sheaves, since the latter is in particular cartesian closed and has epi/mono factorizations (see Goguen [4]). For the "uniqueness of canonical realizations" results, one can work on the sheaf category and then descend to the (full) subcategory $\boldsymbol{P L}$. In fact, observable and bounded-time reachable realizations over $\boldsymbol{P L}$ are also canonical over the larger category because the embedding preserves finite coproducts and limits. Other applications to system theory of the $\boldsymbol{P L}$ concepts introduced in this paper are given in Sontag [8].

3. The objects of $P \boldsymbol{P L}$. The main purpose of this section is to obtain a classification of the isomorphism classes of PL-sets. This classification is of course not as simple as that for the subcategories of finite sets and of finite-dimensional vector spaces, but is nonetheless easy to understand after introducing the proper algebraic structure. The theory will be developed through a series of technical remarks.

A. Ranks and labels. For each fixed $n, D_{n}$ is the set of all finite disjoint families of open polyhedra contained in $\boldsymbol{R}^{n}$. The following defines an equivalence relation in $\boldsymbol{D}_{n}$ :

$$
D E_{1} D^{\prime} \text { iff } \cup\{P, P \text { in } D\}=\cup\left\{Q, Q \text { in } D^{\prime}\right\} .
$$

The relation $E_{0}$ is defined as follows: $D E_{0} D^{\prime}$ is and only if $D=$ 
$\left\{P_{1}, \cdots, P_{r}\right\}$ and there is some hyperplane $H$ intersecting $P_{r}$ such that $D^{\prime}=\left\{P_{1}, \cdots, P_{r-1}, P_{r}^{0}, P_{r}^{+}, P_{r}^{-}\right\}$, where $P_{r}^{0}, P_{r}^{+}$, and $P_{r}^{-}$denote the intersection of $P_{r}$ with $H$ and with the two half-spaces associated to $H$.

LEMma 3.2. The smallest equivalence relation on $\boldsymbol{D}_{n}$ containing $E_{0}$ is $E_{1}$. In fact, $E_{1}$ is the union, over all $i, j$, of the relations $E_{0}^{i} \circ E_{0}^{-j}$.

Proof. Since $E_{1}$ contains $E_{0}$, we need only to prove that, if $D=\left\{P_{1}, \cdots, P_{r}\right\}$ and $D^{\prime}=\left\{Q_{1}, \cdots, Q_{s}\right\}$ have the same union, then there exists a third family $D^{\prime \prime}$ of open polyhedra $\left\{L_{1}, \cdots, L_{t}\right\}$ such that $D E_{0}^{i} D^{\prime \prime}$ and $D E_{0}^{j} D^{\prime \prime}$ for some $i, j$. In other words, $D^{\prime \prime}$ must be such that there is a chain $D=D_{0}, D_{1}, \cdots, D_{i}=D^{\prime \prime}$ with $D_{k} E_{0} D_{k+1}$ for all $k$, and similarly for $D^{\prime}$. Let $\left\{H_{\lambda}\right\}$ be a finite family of hyperplanes constructed as follows. For each $P$ in $D$ or in $D^{\prime}$, pick hyperplanes $K_{1}, \cdots, K_{n}$ such that $P$ is the intersection of some of the $K_{j}$ and of open half-spaces associated to the rest of the $K_{j}$; the $H_{\lambda}$ are then obtained by considering all the $K_{j}$ obtained in this way, for $P$ in $D$ and $D^{\prime}$.

Let $D^{\prime \prime}$ be the family of all those minimally-nonempty intersections $T$ of the $H_{\lambda}$ and open half-spaces associated to the $H_{\lambda}$ for which $T$ is included in the union of the sets in $D$ (or $D^{\prime}$ ). These intersections are disjoint, by minimality, so $D^{\prime \prime}$ is in $\boldsymbol{D}_{n}$. Note that $D E_{1} D^{\prime \prime}$. To obtain a chain from $D$ to $D^{\prime \prime}$, let $D:=\left\{P_{1}, \cdots, P_{r}\right\}$, and write each $P_{j}$ as an intersection of the $H_{\lambda}$ and corresponding half-spaces. If any of the $P_{i}$, say $P_{r}$, is not a minimal intersection, there exists an $H_{\lambda}$ inducing a proper subdivision $P_{r}^{+}, P_{r}^{-}, P_{r}^{0}$. Then $D E_{0} D_{1}$, where $D_{1}$ is $\left\{P_{1}, \cdots, P_{r-1}, P_{r}^{0}, P^{+}, P^{-}\right\}$, and the argument can be repeated with $D_{1}$. Eventually one reaches a $D_{i}$ with all $P_{j}$ minimal (so a member of $D^{\prime \prime}$ ). But every $T$ in $D^{\prime \prime}$ appears in $D_{i}$, since $D^{\prime \prime} E_{1} D_{i}$ and every $T$ in $D^{\prime \prime}$ is included in the union of the $P_{j}$ in $D$. So $D_{i}=D^{\prime \prime}$. The same argument gives a chain from $D^{\prime}$ to $D^{\prime \prime}$.

Pairs of integers are naturally ordered by: $(i, j) \leqq\left(i^{\prime}, j^{\prime}\right)$ whenever $i \leqq i^{\prime}$ and $j \leqq j^{\prime}$. With this ordering:

DeFINITION 3.3. The p-rank of a (nonempty) open polyhedron $P$ is

$$
p \text {-rank }(P):=\left(\operatorname{dim} P, \operatorname{dim} 0^{+} P\right) .
$$

The rank $r(X)$ of a nonempty PL-set $X$ is defined as the maximal possible $p$-rank of a polyhedral subobject of $X$ when there is a unique such maximal rank. 
Definition 3.4. The (open or closed) polyhedron $P$ in $\boldsymbol{R}^{n}$ is acute if it is included in the orthant $\boldsymbol{R}_{+}^{n}$.

The above subclass of polyhedra is introduced for purely technical purposes, mainly because the projective equivalence $\alpha$ introduced in (1.3) will permit reducing many questions about acute polyhedra to questions about polytopes (even though $\alpha$ is not a PL-map).

Lemma 3.5. Let $P$ be an acute open polyhedron in $\boldsymbol{R}^{n}$ of p-rank $(n, m)$, with $n \neq 0$, and $H$ be a hyperplane intersecting $P$. Let $P^{0}$, $P^{+}$, and $P^{-}$be the intersections of $P$ with $H$ and its associated open half-spaces, and let $\left(n^{0}, n^{0}\right),\left(n^{+}, n^{+}\right)$, and $\left(n^{-}, m^{-}\right)$denote the respective p-ranks. Then exactly one of the following possibilities holds:

(a) $\left(n^{+}, m^{+}\right)=\left(n^{-}, m^{-}\right)=(n, m)$ and $\left(n^{0}, m^{0}\right)=(n-1, m)$;

(b) $m \geqq 2,\left(n^{+}, m^{+}\right)=\left(n^{-}, m^{-}\right)=(n, m)$, and $\left(n^{0}, m^{0}\right)=(n-1$, $m-1)$;

(c) for some $k<m,\left(n^{+}, m^{+}\right)=(n, m),\left(n^{-}, m^{-}\right)=(n, k)$, and $\left(n^{0}, m^{0}\right)=(n-1, k)$

(d) idem to (c), with + , - reversed.

Proof. We first note that $P^{+}, P^{-}$(and $P^{0}$ ) are all nonempty. This is because $P$ is open in $\boldsymbol{R}^{n}$ and $H$ is the boundary of its associated half-spaces. In fact, $P^{+}$and $P^{-}$are again open, so also of dimension $n$, while $P^{0}$ is relatively open in $H$ and hence of dimension $n-1$. To understand the dimensions "at infinity" $m^{0}, m^{+}, m^{-}$, we consider the projective equivalence $\alpha: R^{n} \rightarrow \Delta_{n}=\Delta$. Let $Q, Q^{+}, Q^{-}$, $Q^{0}, K$ be the images of $P, P^{+}, P^{-}, P^{0}$, and $H \cap \boldsymbol{R}_{+}^{n}$ under this transformation, denoting also by $K$ the span of the above $K$. Let $K_{\infty}$ denote the hyperplane

$$
x_{1}+\cdots+x_{n}=1 .
$$

Since directions of recession of acute polyhedra correspond to points in $K_{\infty}$ with all $x_{i} \geqq 0$, one has for each of $A:=Q, Q^{0}, Q^{+}, Q^{-}$and $A^{\prime}$ : $=P, P^{0}, P^{+}, P^{-}$(and defining the dimension of the empty set as $-1)$ :

$$
\operatorname{dim} 0^{+} A^{\prime}=1+\operatorname{dim}\left(c \ell(A) \cap K_{\infty}\right) .
$$

There are then four cases to consider:

(i) $c \ell(Q) \cap K_{\infty}=\varnothing$. Here $c \iota(A) \cap K_{\infty}$ is empty for all of the above $A$, and hence case (a) holds with $m=0$.

(ii) $c \ell(Q) \cap K_{\infty} \neq \varnothing$ but $K \cap c \ell(Q) \cap K_{\infty}=\varnothing$. In this case, $c \ell\left(Q^{0}\right) \cap K_{\infty}=c \ell\left(K \cap Q \cap K_{\infty}\right)=K \cap c \ell(Q) \cap K_{\infty}=\varnothing$, so one of $c \ell\left(Q^{+}\right) \cap K_{\infty}$ or $c \ell\left(Q^{-}\right) \cap K_{\infty}$ must also be empty (otherwise a line in 
$K_{\infty}$ joining points in both of the latter would intersect $\left.c \ell\left(Q^{0}\right) \cap K_{\infty}\right)$. If $c \ell\left(Q^{-}\right) \cap K_{\infty}$ is empty, then $c \ell\left(Q^{+}\right) \cap K_{\infty}=c \ell(Q) \cap K_{\infty}$, and (c) holds with $k=0$. Otherwise, (d) holds with $k=0$.

(iii) $K \cap c \ell(Q) \cap K_{\infty} \neq \varnothing$ and $c \ell(Q) \cap K_{\infty}$ is contained in $K \cap K_{\infty}$. In this case all $c \ell(A) \cap K_{\infty}$ are equal, so (a) holds.

(iv) As above but with $c \ell(Q) \cap K_{\infty}$ not contained in $K \cap K_{\infty}$. Necessarily $c \ell(Q) \cap K_{\infty}$ has more than one point so (since a polytope) dimension at least one, and hence $m \geqq 2$. Here $K \cap K_{\infty}$ is a hyperplane of $K_{\infty}$ intersecting the polytope $c \ell(Q) \cap K_{\infty}$ at $c \ell\left(Q^{0}\right) \cap K_{\infty}$. Let $k$ be the dimension of the latter. If $K \cap K_{\infty}$ intersects $Q \cap K_{\infty}$ then $k=m$ and (b) holds. If $K \cap K^{\infty}$ intersects only the boundary then $k<m$ and either $c \ell\left(Q^{\circ}\right) \cap K_{\infty}=c \ell\left(Q^{-}\right) \cap K_{\infty}$ and (c) holds, or $c \ell\left(Q^{0}\right) \cap K_{\infty}=c \ell\left(Q^{+}\right) \cap K_{\infty}$ and (d) holds instead.

DEFINITION 3.7. The $(n, m)$-simplex $s(n, m)$ is the (generalized) open $n$-simplex opconv $(S, D)$, where $S$ is the set of points $0, e_{1}, \cdots, e_{n-m}$ and $D$ is the set of directions of $e_{n-m+1}, \cdots, e_{n}\left(e_{i}\right.$ denotes here the $i$ th canonical vector in $\boldsymbol{R}^{n}$ ).

Thus $s(n, m)$ is an acute polyhedron of $p$-rank $(n, m)$, namely,

$$
\left\{\left(x_{1}, \cdots, x_{n}\right) \text { in } \boldsymbol{R}^{n} \mid \text { all } x_{i}>0 \text { and } x_{1}+\cdots+x_{n-m}<1\right\} \text {, }
$$

(just a point if $n=0$ ) or equivalently, the product $P_{n-m} \times \boldsymbol{R}_{+}^{m}$, where $P_{k}$ is the interior of the standard (bounded) $k$-simplex and $\boldsymbol{R}_{+}$is $(0, \infty)$. Every simplex of $p$-rank $(n, m)$ is isomorphic to $s(n, m)$ under an invertible linear map between their spans.

LeMma 3.9. For open polyhedra the p-rank coincides with the rank.

Proof. We need to show that if there is a one-to-one PL-map $f: Q \rightarrow P$ between open polyhedra then $p$-rank $(Q) \leqq p$-rank $(P)$. For this it will be sufficient to show that the $p$-rank is invariant under isomorphism, since it is clear from the definition that the $p$-rank of a subset of $P$ cannot exceed that of $P$. Assume then that $f$ is bijective.

Consider for each of $P, Q$, the partitions into open subpolyhedra obtained by intersecting with the various orthants of aff $(P)$, aff $(Q)$ and their respective faces (without loss of generality assume aff $(P)=\boldsymbol{R}^{s}$, aff $\left.(Q)=\boldsymbol{R}^{t}\right)$. By (2.4) there exist refinements into open polyhedral partitions $\left\{P_{i}\right\},\left\{Q_{i}\right\}$ such that $f$ is a linear isomorphism in each $Q_{i}$. (By (1.5) we may in fact assume that each element of the partition is a generalized simplex, with corresponding $P_{i}, Q_{i}$ linearly isomorphic.) Since $p$-rank is invariant under linear isomorphism, it will be enough to show that, whenever one has such 
partitions, $p$-rank $(P)=\max$. of the $p$-ranks of the $P_{i}$ (and the same for $Q$ ). If $P$ is acute, this is a consequence of (3.2) and (3.5). In the general case, it will be enough to show that the intersection $P^{\prime}$ of $P$ with at least one of the open orthants of $R^{s}$ has the same rank as $P$. But $0^{+} P$ is open in aff $\left(0^{+} P\right)$ and thus there is some orthant, say $\boldsymbol{R}_{+}^{s}$ under a linear change of coordinates, such that $c \ell\left(\boldsymbol{R}_{+}^{s}\right)$ intersects aff $\left(0^{+} P\right)$ and $0^{+} P$. Thus $P$ recedes in $\operatorname{dim}\left(0^{+} P\right)$ linearly independent directions of vectors in $\boldsymbol{R}_{+}^{s}$ and the corresponding $P^{\prime}$ has equal $p$-rank. One may construct the $p_{i}$ so that some refine $p^{\prime}$, and the result follows from the case of acute polyhedra.

A polyhedron is by (1.5) a disjoint union of (generalized) simplexes. Let

$$
F:=N\left[\left\{s_{i j}, i \geqq j \geqq 0\right\}\right]
$$

be the free abelian monoid on the symbols $s_{i j}$ (here $N$ denotes the nonnegative integers, and elements of $F$ are polynomials in the symbols $s_{i j}$ with coefficients in $N$ and "termwise" addition).

Definition 3.11. An element $a=\sum a_{i j} s_{i j}$ of of $F$ is a label for a PL-set $X$ if there exists a partition of $X$ into open simplexes consisting of precisely $a_{i j}$ simplexes of $\operatorname{rank}(i, j)$.

Of course labels are far from unique. Note that each PL-set has labels and that for each element $a$ of $F$ there is a PL-set $X$ having $a$ as a label; in fact the latter $X$ can be assumed to be in an orthant $\boldsymbol{R}_{+}^{n}$ (one only needs to suitably embed the orthants of an $\boldsymbol{R}^{n-1}$ containing $X$ in disjoint hyperplanes of $\boldsymbol{R}^{n}$ ).

LEMMA 3.12. Two PL-sets are isomorphic if and only if they have a label in common.

Proof. Clear from 2.4 and the previous remarks.

LeMma 3.13. Let $P$ be an open polyhedron. If $\{P\} E_{0}^{i}\left\{Q_{1}, Q_{2}, \cdots\right\}$ and each $Q_{i}$ is isomorphic to a (generalized) simplex, then $P$ is also isomorphic to a simplex.

Proof. We must prove that if $P^{+}, P^{-}, P^{0}$ are all isomorphic to generalized simplices then $P$ also is. Since $s(n, m)=P_{n-m} \times \boldsymbol{R}_{+}^{m}$, this is by 3.5 equivalent to showing that the coproducts of each of:

(a) $P_{k}, P_{k}, P_{k-1}$, 
(b) $\boldsymbol{R}_{+}^{2}, \boldsymbol{R}_{+}^{2}, \boldsymbol{R}_{+}$,

(c) $\boldsymbol{R}_{+}, P_{n}, P_{n-1}, n \geqq 1$,

are all simplices (respectively, $P_{k}, \boldsymbol{R}_{+}^{2}, \boldsymbol{R}_{+}^{n}$ ). The simplex $s(n, m)$ is isomorphic to the cube $I^{n-m} \times \boldsymbol{R}_{+}^{m}$, where $I$ is the open interval $(0,1)$. This is proved in the first paragraph of (3.14) below, and will be used here. Since $I$ is isomorphic to the coproduct of two I's and a point, case (a) follows by multiplication by $I^{k-1}$. Case (b) follows by slicing $P=\boldsymbol{R}_{+}^{2}$ by the hyperplane $K=\left\{x_{1}-x_{2}=0\right\}$ : each of $P^{+}, P^{-}$is a simplex (isomorphic to) $R_{+}^{2}$, and $P_{0}$ is a half-line. We now study case (c). Consider $P=\boldsymbol{R}_{+}^{n}$ and let $K_{\infty}$ be the hyperplane $\left\{x_{1}+\cdots+x_{n}=1\right\}$. Let $P$ - be the bounded component. Then $P^{-}$is isomorphic to $P_{n}$, and $P_{0}$ is $P_{n-1}$. It will be enough then to prove that $P^{+}$is isomorphic to $\boldsymbol{R}_{+}^{n}$. For this, define $f: P^{+} \rightarrow \boldsymbol{R}_{+}^{n}$ as the identity if $x_{1}+\cdots+x_{n-1} \geqq 1$, and $\left(x_{1}, \cdots, x_{n-1}, x_{1}+\cdots+x_{n}-1\right)$ if $x_{1}+\cdots+x_{n-1}<1$; this is a PL-isomorphism.

Proposition 3.14. Every acute open polyhedron is isomorphic to a (generalized) open simplex.

Proof. It is proved in combinatorial topology that a closed polytope is isomorphic to a closed simplex, with the relative interiors mapping to each other, via a continuous PL-map; see for instance Zeeman [9, Lemma 8]. Recalling the form of $s(n, m)$, this implies that any product $X \times \boldsymbol{R}_{+}^{m}$, with $X$ a polytope, is isomorphic to a simplex, so the cubes $I^{i} \times \boldsymbol{R}_{+}^{j}$ used in 3.13 are indeed isomorphic to simplices.

For a general acute polyhedron $P$ the result will be proved by a refinement of the argument for polytopes, using the projective equivalence $\alpha$. "Slicing" first by a suitable hyperplane $x_{1}+\cdots+$ $x_{n}=a$, it is enough by 3.13 to prove the result for a $P$ such that $\alpha(P)$ is in any desired neighborhood of $K_{\infty}$ (notation as in 3.5). A further linear transformation on $P$ can be used to insure that $\alpha(P)$ is in fact contained in a neighborhood of any given point $x$ in $K_{\infty}$ with all $x_{i}>0$. For $\alpha(P)$ small enough, there exists then a simplex $Q$ such that (i) $\alpha(Q)$ contains and has the same dimension as $\alpha(P)$, and (ii) $Q_{\infty}=c \ell(\alpha(Q)) \cap K_{\infty}$ has the same dimension as $P_{\infty}=c \ell(\alpha(P)) \cap K_{\infty}$.

Pick now a point $z$ in the relative interior of the face $P_{\infty}$ of $\alpha(P)$. Define a "pseudo-radial projection" into $z$, as in Zeeman [9, Lemma 8], from a cell subdivision of the boundary of $\alpha(Q)$ into one for the boundary of $\alpha(P)$. Since $z$ is in $K_{\infty}$ and $P_{\infty}, Q_{\infty}$ have equal dimensions, this means joining to $z$ that $\alpha(P)$ and $\alpha(Q)$ admit simultaneous partitions into isomorphic open simplexes $\alpha\left(P_{i}\right), \alpha\left(Q_{i}\right)$ with $\left(P_{i}\right)_{\infty}$ and $\left(Q_{i}\right)_{\infty}$ of equal dimensions. So $P_{i}, Q_{i}$ have the same 
rank and are isomorphic to each other, for each $i$. Thus $P$ is isomorphic to $Q$, as wanted.

B. Classification. We consider now the set $\mathrm{PL}_{0}$ of isomorphism classes of PL-sets, as an abelian monoid, with coproduct as the binary operation and whose identity is the empty set. $\mathrm{By} 1.5, \mathrm{PL}_{0}$ is generated by the generalized simplices. Thus the monoid homomorphism

$$
\lambda: F \longrightarrow \mathrm{PL}_{0}
$$

induced by

$$
\lambda\left(s_{i j}\right):=\text { isoclass of } s(i, j), \quad i \geqq j \geqq 0,
$$

is surjective.

Proposition 3.17. As a monoid congruence, the kernel of $\lambda$ is generated by the following set of equations:

(a) $s_{i j}=2 s_{i j}+s_{i-1, j}, i \geqq 1, j \geqq 0$,

(b) $s_{i j}=2 s_{i j}+s_{i-1, j-1}, i \geqq j \geqq 2$, and

(c) $s_{i j}=s_{i j}+s_{i k}+s_{i-1, k}, j \geqq k, i-1 \geqq k \geqq 0$.

Proof. Each of the above equations is in the kernel of $\lambda$, since they can be realized by slicing the simplex $s(i, j)$ by appropriate hyperplanes, as in the proof of 3.13. Conversely, assume that

$$
\lambda(a)=\lambda(b) .
$$

We want to prove that there exists a sequence of elements of $F$, all mapping into the same isoclass, and such that each of these elements is obtained from the preceeding or succeeding one by one of the above types of substitutions.

Let $X$ and $Y$ be PL-sets in $\boldsymbol{R}_{+}^{n}$ with labels $a$ and $b$ respectively. Since $\lambda(a)=\lambda(b), X$ and $Y$ have by 3.12 a label in common, say $c$. It is enough to prove that $a$ (and hence by the same argument b) is equivalent to $c$ using the above transformations. Let $D$ be a partition of $X$ that gives the label $c$. Then $\{X\} E_{0}^{i} D_{1} E_{0}^{i} D_{2} \cdots E_{0}^{i} D$, with each $i=+1$ or -1 . Each $D_{i}$ has a label $c_{i}$ with $\lambda\left(c_{i}\right)=\lambda\left(c_{i+1}\right)$. Since the polyhedra $D_{i}$ are all acute, 3.5 can be applied at each step, with each of the cases (a), (b), (c) resulting from the corresponding cases in 3.5 .

One has then an algebraic representation for the isoclasses of $\mathrm{PL}$, as elements of the quotient monoid $F /(\operatorname{ker} \lambda)$. Given a PL-set, a label for it can be obtained immediately from any partition of $F$ 
into open simplices, or more generally, (by 3.14), from any partition into open polyhedra contained in orthants.

Matters can be further simplified by the introduction of the two-generator abelian semiring with identity:

$$
N[x, y]=\left\{\sum a_{i j} x_{i} y_{j}, a_{i j} \geqq 0\right\} .
$$

Additively, this is just again $F$, when one identifies

$$
s_{i j}=x^{i-j} y^{j} .
$$

The map $\lambda$, thought of now as defined on $N[x, y]$, is a semiring homomorphism when $\mathrm{PL}_{0}$ is viewed as a semiring using (cartesian, or categorical) product as multiplication. The multiplicative identity 1 is the one-point set, $\lambda(x)$ is the open unit interval $I=(0,1)$, and $\lambda(y)$ is $\boldsymbol{R}_{+}$. The semiring structure permits giving a finite presentation for $\mathrm{PL}_{0}$ :

THEOREM 3.21. Let $\mathrm{PL}_{0}$ be the set of isomorphism classes of objects of PL, thought of as a semiring with coproduct as addition and product as multiplication. Then $\mathrm{PL}_{0}$ is isomorphic to the semiring

$$
S:=N[x, y] / E,
$$

where $E$ is the semiring congruence generated by the three equations

$$
\begin{aligned}
& \text { (a) } x=2 x+1 \\
& \text { (b) } y^{2}=2 y^{2}+y, \\
& \text { (c) } y=x+y+1 .
\end{aligned}
$$

Proof. In terms of $x$ and $y$, a set of equations generating $E=$ $\operatorname{ker} \lambda$ is known to be, from (4.17),

(a) $x^{n} y^{m}=2 x^{n} y^{m}+x^{n-1} y^{m}, \quad n \geqq 1, \quad m \geqq 0$,

(b) $x^{n} y^{m}=2 x^{n} y^{m}+x^{n} y^{m-1}, \quad n \geqq 0, \quad m \geqq 2$,

(c) $x^{d} y^{k+t}=x^{d} y^{k+t}+x^{d+t} y^{k}+x^{d+t-1} y^{k}$,

$$
d \geqq 0, \quad t \geqq 0, \quad k \geqq 0, \quad d+t \geqq 1 .
$$

As generators of a semiring congruence, these equations are in turn equivalent to the simpler set consisting of (3.23a), (3.23b) and

$$
y^{t}=y^{t}+x^{t}+x^{t-1}, \quad t \geqq 1 .
$$

Since $(3.23 \mathrm{c})$ is the particular case $t=1$ of this, the proof will be complete once that we establish that (3.25) follows from (3.23). By induction assume that (3.25) is true for $t-1(t \geqq 2)$; then: 


$$
\begin{aligned}
y^{t} & =y^{t-2} y^{2}=y^{t-2}\left(2 y^{2}+y\right) \\
& =2 y^{2}+y^{t-1} \\
& =2 y^{t-1}(x+y+1)+y^{t-1} \\
& =\left(2 y^{t}+3 y^{t-1}+x y^{t-1}\right)+x y^{t-1} \\
& =\left(2 y^{t}+3 y^{t-1}+x y^{t-1}\right)+x\left(y^{t-1}+x^{t-1}+x^{t-2}\right) \\
& =\left(2 y^{t}+2 x y^{t-1}+3 y^{t-1}\right)+\left(x^{t}+x^{t-1}\right),
\end{aligned}
$$

and we need to show that the first of these is again $y^{t}$. But this term is

$$
\begin{aligned}
y^{t-2}\left[\left(2 y^{2}+y\right)+2 y+2 x y\right] & =y^{t-2}\left(y^{2}+2 y+2 x y\right) \\
& =y^{t-1}[y+1+(1+2 x)] \\
& =y^{t-1}(y+1+x) \\
& =y^{t} .
\end{aligned}
$$

Let

$$
\hat{\chi}: \text { PL-sets } \longrightarrow S
$$

be the characteristic of $P \boldsymbol{L}$, the map assigning to each PL-set its equivalence class of labels. This is a useful object to consider when answering questions about the category. Note that $\hat{\chi}$ can be used to translate into purely algebraic problems even questions not exclusively about isomorphism. For instance, asking whether there is an epimorphism $f: X \rightarrow Y$ between PL-sets amounts by the results of $\S 3$ to asking if $Y$ is a subobject of $X$, and this is in turn equivalent to deciding if $\hat{\chi}(Y)$ is a summand of $\hat{\chi}(X)$ in $S$. Similarly, $Y$ is a factor of $X$ ( $f$ "splits") if and only if $\hat{\chi}(Y)$ divides $\hat{\chi}(X)$. As an example, we solve here the epi/mono question for the case of well-defined ranks (the solution in the general case follows easily from this).

Proposition 3.27. Let $X, Y$ be PL-sets with well-defined ranks. Then there exists a monomorphism $f: X \rightarrow Y$ if and only if either one of the following conditions hold:

(i) $\operatorname{dim} X>1$ and $r(X) \leqq r(Y)$.

(ii) $\operatorname{dim} X=\operatorname{dim} Y=1$ and the coefficient of $y$ in a label of $X$ never exceeds that of $y$ in a label of $Y$.

(iii) $\operatorname{dim} Y>\operatorname{dim} X=1$ and $r(X) \leqq r(Y)$.

(iv) $X$ is finite and $\operatorname{card}(X) \leqq \operatorname{card}(Y)$.

Proof. The condition $r(X) \leqq r(Y)$ is necessary, by definition of rank. Assume now that $\operatorname{dim} X=n+m>1$ and let $r(X)=(n+m, m) \leqq$ $(u+v, v)=r(Y)$. Let $a, b$ be labels of $X, Y$. Each monomial $x^{i} y^{j}$ 
is a summand of $x^{n} y^{m}$, and hence of $x^{u} y^{v}$, so $a$ is a summand of $r x^{u} y^{v}$ for some integer $r$. Iterating (3.24a), the latter is in fact a summand of a single monomial $x^{u} y^{v}$. But $b$ contains one such monomial, since $r(Y)=(u+v, v)$. Thus $a$ is a summand of $b$, and (i) is proved. When the dimension of $X$ is 1 , the possibilities are $r(X)=(1,0)$ or $(1,1)$. In the first case, $a=\alpha x+\beta$ is a summand of $x$ and hence of $b$. In the second case, $a=\alpha x+\beta y+\gamma$, which is a summand of a suitable $k y$. Since $r(Y) \geqq(2,1), b$ has $a$ term $x y$. By (3.24a), this admits a summand $k x y$, which by (3.23a) has a summand $k y$. Thus (ii) follows. To prove (iii), note that $a=\alpha x+\beta y+\gamma, b=$ $\alpha^{\prime} x+\beta^{\prime} y+\gamma^{\prime}, \alpha \alpha^{\prime} \neq 0, \beta^{\prime} \geqq \beta$. By (3.23a), $\alpha x+\gamma$ is a summand of $x$, hence of $\alpha^{\prime} x$. And $\beta y$ is a summand of $\beta^{\prime} y$, so $a$ is a summand of $b$, as wanted.

It is interesting to remark that the classical theorem of Euler on counting faces of polyhedra is a consequence of the form of $S$. Let

$$
\chi: \text { PL-sets } \longrightarrow N
$$

be obtained by composing $\hat{\chi}$ with the evaluation $x:=-1, y:=0$ (well-defined by the form of $E$ ). In terms of $S$, a PL-set $X$ is isomorphic to an acute polyhedron iff it is isomorphic to a cube, i.e., of the form $\lambda\left(x^{i} y^{j}\right)$. In particular, an open polytope of dimension $n$ has label $x^{n}$. Thus $\hat{\chi}(X)=(-1)^{n}$. A closed polytope $P$ is (see proof of 3.14) isomorphic to a closed cube, i.e., it has a label $(x+2)^{n}$, so $\hat{\chi}(P)=1$ always. Writing a closed polytope $P$ as a disjoint union of the relative interiors of its faces, one has, if $P$ has $d_{i}$ faces of dimension $i$, that $\sum_{i=0}^{\infty}(-1)^{i} d_{i}=1$ (Euler's theorem). The map $\chi$ can be extended to $\psi$ : PL-sets $\rightarrow Z^{2}$, by evaluation at $x:=(-1,-1)$, $y:=(0,-1)$. This map is universal for groups, and establishes $\boldsymbol{Z}^{2}$ as the Grothendieck group of PL. Note that by studying the free abelian monoid (rather than group) generated by the open simplexes, one obtains a complete characterization of isoclasses, from which the Grothendieck group can be in turn derived.

We turn now to the word problem for $S$, i.e., deciding for given $a, b$ in $N[x, y]$ if they are equivalent under $E$. Since the generators of $E$ all preserve degree, it is clear that in deciding if $a E b$ one may restrict attention to the free abelian monoid

$$
F_{n}:=N\left[\left\{s_{i j} \mid 0 \leqq j \leqq i \leqq n\right\}\right],
$$

where $n$ is the dimension of $\lambda(a)$ and $\lambda(b)$, modulo the congruence generated by those equations in (3.15) which involve only $i \leqq n$. Note that $F_{n}$ is just the product monoid $N^{2 n}$. 
There are a considerable number of results each of which implies the decidability of word problems in a monoid $M=N^{2 n}$. For example, one may use the results on integer equations given by Ginsburg and Spanier [3]. Another approach is to note that every monoid congruence on the (finitely generated commutative) monoid $M$ is a rational subset of $M \times M$, as proved in Filenberg and Schützenberger [2] (in fact, they also prove that there are always rational cross-sections for such congruences). A rational subset of $M \times M$ is recognizable by a generalized finite automaton (Eilenberg [2, Theorem VII. 10.1]). Thus checking if $a E b$ is equivalent to checking if $(a, b)$ is accepted by a given automaton, a purely algorithmic process.

To rigorously state the implications of the above one would need to give a precise meaning to the phase "given a PL-set". This could mean for example "given by a sentence in the language $L$ " introduced in (2.5), restricting unary operators and constants to computable real numbers. Obtaining a polyhedral partition, i.e., a label, becomes a problem in linear algebra. We shall assume in any case that a PL-set is "given" by specifying a label for it. Decidability of word problems in each $F_{n}$ gives then:

THEOREM 3.30. Isomorphism of PL-sets is decidable.

\section{REFERENCES}

1. M. A. Arbib and E. Manes, Machines in a category: an expository introduction, SIAM Review, 57 (1974), 163-192.

2. S. Eilenberg and M. P. Schützenberger, Rational sets in commutative monoids, J. Algebra, 13 (1969), 173-191.

3. S. Ginsburg and E. H. Spanier, Bounded Algol-like languages, Trans. Amer. Math. Soc., 113 (1964), 333-368.

4. J. A. Goguen, Minimal realizations of machines in closed categories, Bull. Amer. Math. Soc., 78 (1972), 777-783.

5. B. Grünbaum, Convex Polytopes, Wiley, 1967.

6. P. T. Johnstone, Topos Theory, Academic Press, 1977.

7. R. T. Rockaffelar, Convex Analysis, Princeton Univ. Press, 1970.

8. E. D. Sontag, Nonlinear regulation: the piecewise-linear approach, IEEE Trans. Autom. Control, 26 (1981), 346-358.

9. E. C. Zeeman, Seminar on Combinatorial Topology, Publ. I.H.E.S., 1963.

Received November 19, 1979 and in revised form January 22, 1981. Supported in part by U. S. Air Force Grant AFOSR-F49620-79-C-0117.

RUtgers UNIVERSity

New Brunswick, NJ 08903 



\title{
PACIFIC JOURNAL OF MATHEMATICS
}

\section{EDITORS}

\author{
DONALD BABBITT (Managing Editor) \\ University of California \\ Los Angeles, CA 90024 \\ Hugo RossI \\ University of Utah \\ Salt Lake City, UT 84112 \\ C. C. Moore and ANDrew OGG \\ University of California \\ Berkeley, CA 94720
}

\section{J. DugundjI}

Department of Mathematics

University of Southern California

Los Angeles, CA 90007

R. FinN and J. Milgram

Stanford University

Stanford, CA 94305

\section{ASSOCIATE EDITORS}
R. ARENS
E. F. BeCKENBACH
B. H. NeUMANN
F. WOLF
K. YoshidA

\section{SUPPORTING INSTITUTIONS}

UNIVERSITY OF ARIZONA

UNIVERSITY OF BRITISH COLUMBIA

CALIFORNIA INSTITUTE OF TECHNOLOGY

UNIVERSITY OF CALIFORNIA

MONTANA STATE UNIVERSITY

UNIVERSITY OF NEVADA, RENO

NEW MEXICO STATE UNIVERSITY

OREGON STATE UNIVERSITY

\author{
UNIVERSITY OF OREGON \\ UNIVERSITY OF SOUTHERN CALIFORNIA \\ STANFORD UNIVERSITY \\ UNIVERSITY OF HAWAII \\ UNIVERSITY OF TOKYO \\ UNIVERSITY OF UTAH \\ WASHINGTON STATE UNIVERSITY \\ UNIVERSITY OF WASHINGTON
}

The Supporting Institutions listed above contribute to the cost of publication of this Journal, but they are not owners or publishers and have no responsibility for its content or policies.

Mathematical papers intended for publication in the Pacific Journal of Mathematics should be in typed form or offset-reproduced, (not dittoed), double spaced with large margins. Please do not use built up fractions in the text of the manuscript. However, you may use them in the displayed equations. Underline Greek letters in red, German in green, and script in blue. The first paragraph or two must be capable of being used separately as a synopsis of the entire paper. Please propose a heading for the odd numbered pages of less than 35 characters. Manuscripts, in triplicate, may be sent to any one of the editors. Please classify according to the scheme of Math. Reviews, Index to Vol. 39. Supply name and address of author to whom proofs should be sent. All other communications should be addressed to the managing editor, or Elaine Barth, University of California, Los Angeles, California, 90024.

50 reprints to each author are provided free for each article, only if page charges have been substantially paid. Additional copies may be obtained at cost in multiples of 50 .

The Pacific Journal of Mathematics is issued monthly as of January 1966. Regular subscription rate: $\$ 102.00$ a year (6 Vols., 12 issues). Special rate: $\$ 51.00$ a year to individual members of supporting institutions.

Subscriptions, orders for numbers issued in the last three calendar years, and changes of address shoud be sent to Pacific Journal of Mathematics, P.O. Box 969, Carmel Valley, CA 93924, U.S.A. Old back numbers obtainable from Kraus Periodicals Co., Route 100, Millwood, NY 10546.

PUBLISHED BY PACIFIC JOURNAL OF MATHEMATICS, A NON-PROFIT CORPORATION

Printed at Kokusai Bunken Insatsusha (International Academic Printing Co., Ltd.). 8-8, 3-chome, Takadanobaba, Shinjuku-ku, Tokyo 160, Japan. 


\section{Pacific Journal of Mathematics}

\section{Vol. 98, No. 1 \\ March, 1982}

Humberto Raul Alagia, Cartan subalgebras of Banach-Lie algebras of

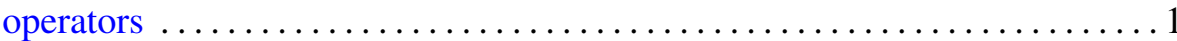

Tom M. (Mike) Apostol and Thiennu H. Vu, Elementary proofs of

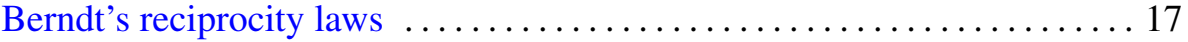

James Robert Boone, A note on linearly ordered net spaces $\ldots \ldots \ldots \ldots . \ldots 25$

Miriam Cohen, A Morita context related to finite automorphism groups of

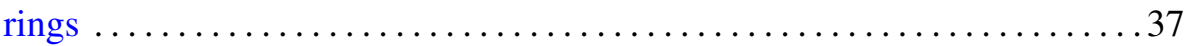

Willibald Doeringer, Exceptional values of differential polynomials . . . . . . 55

Alan Stewart Dow and Ortwin Joachim Martin Forster, Absolute

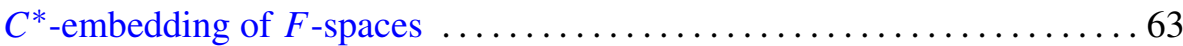

Patrick Hudson Flinn, A characterization of $M$-ideals in $B\left(l_{p}\right)$ for

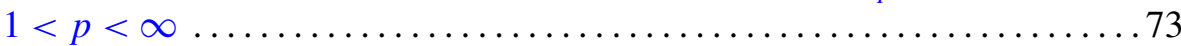

Jack Emile Girolo, Approximating compact sets in normed linear spaces . . . 81

Antonio Granata, A geometric characterization of $n$th order convex

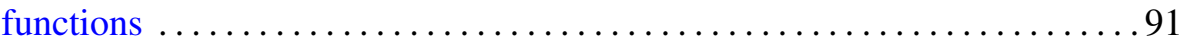

Kenneth Richard Johnson, A reciprocity law for Ramanujan sums . . . . . . .99

Grigori Abramovich Kolesnik, On the order of $\zeta\left(\frac{1}{2}+i t\right)$ and $\Delta(R) \ldots \ldots 107$

Daniel Joseph Madden and William Yslas Vélez, Polynomials that

represent quadratic residues at primitive roots $\ldots \ldots \ldots \ldots \ldots \ldots \ldots 123$

Ernest A. Michael, On maps related to $\sigma$-locally finite and $\sigma$-discrete

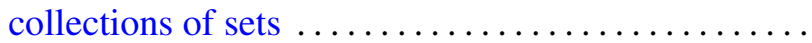

Jean-Pierre Rosay, Un exemple d'ouvert borné de $\mathbf{C}^{3}$ “taut" mais non

hyperbolique complet

Roger Sherwood Schlafly, Universal connections: the local problem

Russel A. Smucker, Quasidiagonal weighted shifts .....

Eduardo Daniel Sontag, Remarks on piecewise-linear algebra . .

Jan Søreng, Symmetric shift registers. II

H. M. (Hari Mohan) Srivastava, Some biorthogonal polynomials suggested

by the Laguerre polynomials 